\title{
Heat Capacity of the Cerium and Ytterbium Orthovanadates
}

\section{Liubov T. Denisova and Liubov G. Chumilina* \\ Siberian Federal University \\ 79 Svobodny, Krasnoyarsk, 660041 Russia}

Received 17.04.2015, received in revised form 04.05.2015, accepted 07.06.2015

The cerium and ytterbium orthovanadates were synthesized by a solid-phase method. The temperature dependence of the molar heat capacity of oxidation compounds was measured by differential scanning calorimetry.

Keywords: the cerium and ytterbium orthovanadates, heat capacity, thermodynamic properties.

DOI: $10.17516 / 1998-2836-2015-8-2-306-311$.

\section{Теплоемкость ортованадатов}

церия и иттербия

Л.Т. Денисова, Л.Г. Чумилина

Сибирский федеральный университет Россия, 660041, Красноярск, пр. Свободный, 79

Твердофазным методом синтезированы ортованадаты иерия и иттербия. Методом дифференцильной сканирующей калориметрии исследовано влияние температуры на молярную теплоемкость оксидных соединений.

Ключевые слова: ортованадаты иерия и иттербия, теплоемкость, термодинамические свойства.

(C) Siberian Federal University. All rights reserved

* Corresponding author E-mail address: antluba@mail.ru 


\section{Введение}

Кристаллы редкоземельных ортованадатов $\mathrm{RVO}_{4}(\mathrm{R}=\mathrm{La}-\mathrm{Lu})$ обладают целым рядом свойств, позволяющих использовать их в науке, технике, медицине [1-5]. Несмотря на такое внимание к подобным соединениям, данные о их термодинамических свойствах при высоких температурах (в первую очередь это касается $\mathrm{CeVO}_{4}$ и $\mathrm{YbVO}_{4}$ ) отсутствуют. В то же время для нахождения оптимальных условий синтеза ортованадатов редкоземельных элементов (РЗЭ), уточнения фазовых равновесий методами термодинамики требуются сведения об их термодинамических свойствах.

В работе [6] проанализирована связь удельной теплоемкости $C_{p}^{o}$ редкоземельных ортованадатов с радиусом иона $\mathrm{R}^{3+}$. При этом показано, что значения $C_{p}^{o}$ закономерно изменяются в зависимости от радиуса $\mathrm{R}^{3-}$ в пределах соответствующих тетрад (La-Nd, Pm-Gd, Gd-Ho, Er-Lu). Тем не менее данные по удельной теплоемкости $\mathrm{CeVO}_{4}$ и $\mathrm{YbVO}_{4}$ отсутствуют.

Целью настоящей работы является исследование температурной зависимости молярной теплоемкости $C_{p}$ ортованадатов $\mathrm{CeVO}_{4}$ и $\mathrm{YbVO}_{4}$.

\section{Экспериментальная часть}

Соединения $\mathrm{CeVO}_{4}$ и $\mathrm{YbVO}_{4}$ получали по керамической технологии с подбором оптимальных режимов синтеза и спекания. Синтез проводили из $\mathrm{CeO}_{2}$ (ос.ч), $\mathrm{V}_{2} \mathrm{O}_{5}$ (ос.ч) и $\mathrm{Yb}_{2} \mathrm{O}_{3}$ (хч). Исходные оксиды предварительно прокаливали на воздухе при $1173 \mathrm{~K}\left(\mathrm{CeO}_{2}, \mathrm{Yb}_{2} \mathrm{O}_{3}\right)$ и $973 \mathrm{~K}\left(\mathrm{~V}_{2} \mathrm{O}_{5}\right)$. После тщательного перемешивания в агатовой ступке смесь прессовали в таблетки, которые обжигали на воздухе последовательно при температурах 873, 893, 913, 933, 953 К (по 15 ч), 973, 1073, 1173, 1273 К (по 10 ч). Контроль фазового состава синтезированных соединений проводили с использованием рентгенофазового анализа (дифрактометр X $\square$ Pert Pro MPD фирмы PANalytical) на излучении $\mathrm{CuK}_{\alpha}$. Регистрация выполнялась высокоскоростным детектором PIXcel с графитовым монохроматором в интервале углов 17-130 с шагом $0.013^{\circ}$. Параметры решетки определены путем полнопрофильного уточнения методом минимизации производной разности [7]. Эти значения для $\mathrm{CeVO}_{4}$ (пр. гр. I4/ $/ \mathrm{amd} ; \mathrm{V}=356.07(2) \AA^{3}$ ) и $\mathrm{YbVO}_{4}$ (пр. гр. $\mathrm{I}_{1} / \mathrm{amd} ; \mathrm{V}=309.998(6) \AA^{3}$ ) в сравнении с результатами других авторов приведены в таблице. Можно заключить, что получены достаточно близкие значения параметров элементарных ячеек.

Измерения теплоемкости осуществлены в платиновых тиглях на приборе STA 449 C Jupiter (NETZSCH). При этом использовали специальные держатели для измерения теплоемкости TGDSC 6.226.1-72+S. Методика измерений подобна описанной в работе $[15,16]$. Экспериментальные данные обрабатывали с помощью пакета анализа NETZSCH Proteus Thermal Analysis и лицензионного программного инструмента Systat Sigma Plot 12.

\section{Результаты и обсуждение}

Экспериментальные результаты по влиянию температуры на $C_{p}$ ортованадатов $\mathrm{CeVO}_{4}$ и $\mathrm{YbVO}_{4}$ показаны на рисунке.

Из этих данных видно, что в исследованных интервалах температур значения $C_{p}$ для этих оксидных соединений закономерно увеличиваются, а полученные зависимости $C_{p}=f(T)$ могут быть описаны классическим уравнением Майера-Келли

$$
-307-
$$


Таблица. Параметры элементарных ячеек $\mathrm{CeVO}_{4}$ и $\mathrm{YbVO}_{4}$

\begin{tabular}{|c|c|c|c|}
\hline$a, \AA$ & $c, \AA$ & $c / a$ & Источник \\
\hline \multicolumn{4}{|c|}{$\mathrm{CeVO}_{4}$} \\
\hline 7.4013 & 6.4980 & 0.878 & [1] \\
\hline 7.3574 & 6.4668 & 0.879 & [8] \\
\hline $7.3668(5)$ & $6.4852(5)$ & 0.880 & [9] \\
\hline $7.4004(2)$ & $6.4972(1)$ & 0.878 & [10] \\
\hline $7.400(1)$ & $6.495(1)$ & 0.878 & [11] \\
\hline 7.4004(1) & $6.4982(6)$ & 0.878 & [12] \\
\hline 7.4016(1) & $6.4980(1)$ & 0.878 & [13] \\
\hline $7.4023(2)$ & $6.4983(2)$ & 0.878 & Наши данные \\
\hline \multicolumn{4}{|c|}{$\mathrm{YbVO}_{4}$} \\
\hline $7.0427(1)$ & $6.2472(1)$ & 0.887 & [10] \\
\hline $7.043(1)$ & $6.242(1)$ & 0.886 & [14] \\
\hline $7.04381(6)$ & $6.24804(7)$ & 0.887 & Наши данные \\
\hline
\end{tabular}

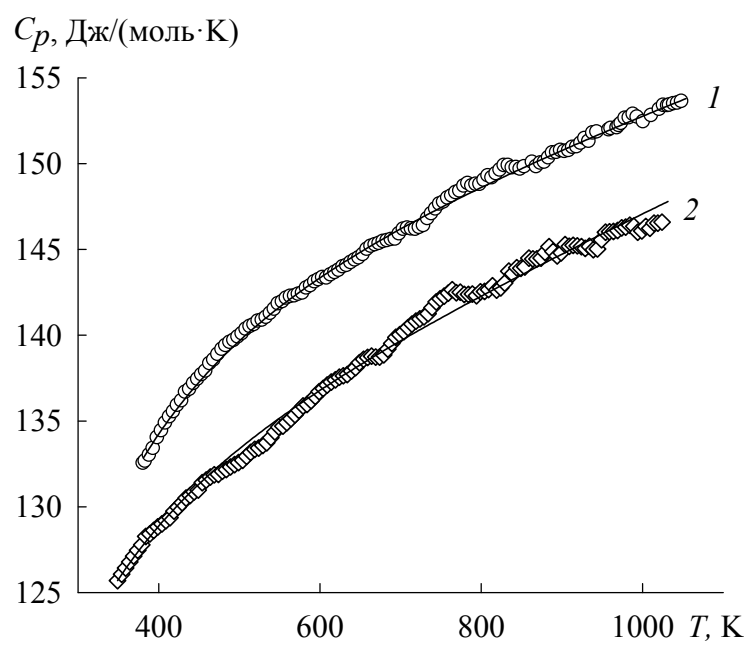

Рис. Влияние температуры на теплоемкость $\mathrm{YbVO}_{4}(1)$ и $\mathrm{CeVO}_{4}$ (2): точки - экспериментальные данные, линия - аппроксимирующая кривая. Данные для $\mathrm{YbVO}_{4}$ смещены на 5 Дж/(моль К) относительно $\mathrm{CeVO}_{4}$

$$
C_{p}=a+b T+c T^{-2}
$$

которое для $\mathrm{CeVO}_{4}$ и $\mathrm{YbVO}_{4}$ имеет, соответственно, следующий вид:

$$
\begin{aligned}
& C_{p}=(127.20 \pm 0.40)+(20.9 \pm 0.4) \cdot 10^{3} T-(10.74 \pm 0.46) \cdot 10^{5} T^{-2} . \\
& C_{p}=(133.48 \pm 0.25)+(16.0 \pm 0.2) \cdot 10^{3} T-(17.02 \pm 0.31) \cdot 10^{5} T^{-2} .
\end{aligned}
$$

Сравнить полученные значения $C_{p}$ для $\mathrm{CeVO}_{4}$ и $\mathrm{YbVO}_{4}$ с другими данными не представлялось возможным, поскольку таких сведений в литературе нет. В то же время в работе [6] на основании тетрад-эффекта для $\mathrm{CeVO}_{4}$ оценено значение $C_{p}^{o} \approx 0.47$ Дж/(г К). По нашим данным, 
$C_{p}^{o}$ для $\mathrm{CeVO}_{4}$ также равно 0.47 Дж/(г·К). Для $\mathrm{YbVO}_{4}$ значение $C_{p}^{o}=0.41$ Дж/(г·К), что тоже хорошо согласуется с результатами [6] для тетрад-эффекта.

Изучению тетрад-эффекта посвящен целый ряд работ [17-21], в которых свойства соединений РЗЭ представлены в зависимости от их атомного номера. Установлено, что этот эффект наблюдается при распеределении редкоземельных элементов между органической и водной фазами, в природных объектах как нарушение плавной формы спектра нормализованных содержаний РЗЭ [18], в комплексных соединениях РЗЭ [19] и др. Все это мы анализировать не будем, только отметим, что установленная в [6] связь между $C_{p}^{o}$ и $\mathrm{R}^{3+}$ в виде тетрад-эффекта М-типа (выпуклые изгибы [20]) позволяет уточнить имеющиеся данные по удельной теплоемкости ортованадатов РЗЭ (или оценивать неизвестные значения).

Работа выполнена при частичной финансовой поддержке работ, выполняемых в рамках Государственного задания Министерства образования и науки Российской Федерации Сибирскому федеральному университету на 2014-2016 годы (проект № 382: Исследование физико-химических свойств сложных оксидных соединений на основе элементов III - V групп ПС им. Д.И. Менделеева, обладающих нелинейно-оптическими свойствами) и КГАУ «Красноярский краевой фонд поддержки научной и научно-технической деятельности».

\section{Список литературы}

1. Фотиев А.А., Слободин Б.В., Ходос М.Я. Ванадаты. Состав, синтез, структура, свойства. М. Наука, 1988. 272 с. [Fotiev A. A., Slobodin B. V., Khodos M. Ya. Vanadates: Composition, Synthesis, Structure, and Properties. Moscow: Nauka, 1988. 272 p. (in Russian)].

2. Денисова Л.Т., Каргин Ю.Ф., Белоусова Н.В., Денисов В.М. Ортованадаты редкоземельных элементов (Обзор). Ч. 1 // Материаловедение. 2014. № 8. С. 18-24. [Denisova L. T., Kargin Yu. F., Belousova N. V., Denisov V. M. Rare earth orthovanadate (Review). Part $1 / /$ Materialovedenie 2014. № 8. P.18-24. (in Russian)].

3. Каминский А.А. Тетрагональные ванадаты $\mathrm{REVO}_{4}(\mathrm{RE}=\mathrm{Ln}(\mathrm{Ce}-\mathrm{Lu}), \mathrm{Y})$ - новый класс ВКР-активных кристаллов // ДАН. 2013. Т. 450. № 3. С. 279-282 [Kaminskii A.A. Tetragonal vanadates $\mathrm{REVO}_{4}(\mathrm{RE}=\mathrm{Ln}(\mathrm{Ce}-\mathrm{Lu}), \mathrm{Y})$ - a novel glass of SRS- active crystals // Doklady Physics. 2013. T. 58. № 5. C. 165-168].

4. Басиев Т.Т., Осико В.В. Новые материалы для ВКР-лазеров // Успехи химии. 2006. Т. 75. № 10. C. 939-955 [Basiev T.T., Osiko V.V. New materials for SRS lasers // Russian Chemical Reviews. 2006. V. 75. № 10. P. 847-862].

5. Патент RU № 2234349. Перельман М.И., Ночевник М.Н., Стрельцов В.П., Рившин М.М., Семенов Г.И., Сироткин А.А. Способ лечения гнойно-воспалительных процессов в мягких тканях и внутренних органах с помощью лазерного излучения и установка для его осуществления. Опубл. 20.08.2004. [RF Patent No. 2234349. Perel'man M. I., Nochevnik M. N., Strel'tsov V. P., Rivshin M.M., Semenov G.I., Sirotkin A.A. The method of treatment of purulent-inflammatory processes in soft tissues and internal organs with the aid of laser radiation and device for its implementation. Publ. Date 20.08.2004].

6. Денисова Л.Т., Каргин Ю.Ф., Денисов В.М. Теплоемкость редкоземельных купратов, ортованадатов и алюмо-, гало- и феррогранатов // ФТТ. 2015. Т. 57. № 8. C. 1658-1662. [Denisova

$$
-309-
$$


L.T., Kargin Yu. F., Denisov V. M. Heat capacity of rare earth cuprates orthovanadates and aluminium-, gallium- and ferrogarnet // Physic of the Solid State. 2015. V. 57. № 8. P. 1658-1662].

7. Solovyov L.A. Full-profile refinement by derivative difference minimization // J. Appl. Crystallogr. 2004. Vol. 37. P. 743-749.

8. Selvan R.K., Gadanken A., Anikumar P., Manikandan G., Karunakaran C. Synthesis and characterization of rare earth orthovanadate $\left(\mathrm{RVO}_{4} ; \mathrm{R}=\mathrm{La}, \mathrm{Ce}, \mathrm{Nd}, \mathrm{Sm}, \mathrm{Eu} \& \mathrm{Gd}\right)$ nanorods/ nanocrystals/nanospindles by a facile sonochemical method and ther catalytic properties // Journal of Cluster Science 2009. Vol. 20. P. 291-305.

9. Mahapatra S., Madras G., Row T.N.G. Synthesis, characterization and photocatalytic activity of lanthanide (Ce, Pr and Nd) orthovanadates // Industrial \& Engineering Chemistry Research, 2007. Vol. 46. P. 1013-1017.

10. Chakoumakos B.C., Abraham M.M., Boatner L.A. Crystal structure refinements of zircontype $\mathrm{MVO}_{4}(\mathrm{M}=\mathrm{Sc}, \mathrm{Y}, \mathrm{Ce}, \mathrm{Pr}, \mathrm{Nd}, \mathrm{Tb}, \mathrm{Ho}, \mathrm{Er}, \mathrm{Tm}, \mathrm{Lu}) / /$ Journal Solid State Chemistry, 1994. Vol. 109. P. 197-202.

11. Varma S., Wani B.N., Gupta M.M. Synthesis, characterization, and redox behavior of mixed orthovanadates $\mathrm{La}_{1-\mathrm{x}} \mathrm{Ce}_{\mathrm{x}} \mathrm{VO}_{4} / /$ Materials Research Bulletin, 2002. Vol. 37. P. 2117-2127.

12. Petit C.T.G., Lan R., Cowin P.I., Tao S. Structure and conductivity of srontium-doped cerium orthovanadates $\mathrm{Ce}_{1-\mathrm{x}} \mathrm{Sr}_{\mathrm{x}} \mathrm{VO}_{4}(0 \leq \mathrm{x} \leq 0.175)$ // Journal Solid State Chemistry 2010. Vol. 183. P. 12311238 .

13. Watanabe A. Highly conductive oxides, $\mathrm{CeVO}_{4}, \mathrm{Ce}_{1-\mathrm{x}} \mathrm{M}_{\mathrm{x}} \mathrm{VO}_{4-0.5 \mathrm{x}}(\mathrm{M}=\mathrm{Ca}, \mathrm{Sr}, \mathrm{Pb})$ and $\mathrm{Ce}_{1-}$ ${ }_{\mathrm{y}} \mathrm{Bi}_{\mathrm{y}} \mathrm{VO}_{4}$ with zircon-type structure prepared by solid-state reaction in air // Journal Solid State Chemistry 2000. V. 153. P. 174-179.

14. Terada Y., Shimamura K., Fukuda T. Growth and optical properties of RE doped bulk and fiber single crystals by Czochralski and micro pulling down methods // Journal of Alloys and Compounds 1998. V. 275-277. P. 697-701.

15. Денисов В.М., Денисова Л.Т., Иртюго Л.А., Биронт В.С. Теплофизические свойства монокристаллов $\mathrm{Bi}_{4} \mathrm{Ge}_{3} \mathrm{O}_{12}$ // ФТT. 2010. Т. 52. № 7. С. 1274-1277 [Denisov V. M., Denisova L.T., Irtyugo L.A., Biront V. S. Thermal physical properties of $\mathrm{Bi}_{4} \mathrm{Ge}_{3} \mathrm{O}_{12}$ single crystals // Physic of the Solid State. 2010. V. 52. № 7. Р. 1362-1365].

16. Денисова Л.Т., Чумилина Л.Г., Бабицкий Н.А., Жереб В.П., Денисов В.М. Теплоемкость и термодинамические свойства оксидных соединений системы $\mathrm{Bi}_{2} \mathrm{O}_{3}-\mathrm{P}_{2} \mathrm{O}_{5} /$ Журнал СФУ. Химия. 2015. № 1. С. 137-142. [Denisova L.T., Chumilina L.G., Babitskiy N.A., Zhereb V.P., Denisov V. M. High-Temperature Heat Capacity and Thermodynamic Properties of Oxide Compounds of $\mathrm{Bi}_{2} \mathrm{O}_{3}-\mathrm{P}_{2} \mathrm{O}_{5} / /$ Journal of Siberian Federal University. Chemistry. 2015. V.8. № 1. P. 137-142. (in Russian)]

17. Kawabe I. Lanthanide tetrad effect in the $\mathrm{Ln}^{3+}$ ionic radii and refined spin-pairing energy theory // Geochem. J. 1992. V. 26. P. 309-335.

18. Ясныгина Т.А., Рассказов С.В. Редкоземельные спектры с тетрад-эффектом: появление в палеозойских гранитоидах окинской зоны Восточного Саяна // Геохимия. 2008. № 8. С. 877-889. [Yasnygina T. A., Rasskazov S. V. Tetrad effect in rare earth element distribution patterns: Evidence from the Paleozoic granitoids of the Oka zone, Eastern Sayan // Geochemistry International 2008. V. 46 № 8. P. 814-825]. 
19. Джуринский Б.Ф. Периодичность свойств лантаноидов // ЖНХ. 1980. Т. 25. № 1. С. 4146. [Dzhurinskii B.F. The periodicity of properties of lanthanide // Russian Journal of Inorganic Chemistry. 1980. Vol. 25(1). P. 41-46. (in Russian)].

20. Masuda A., Kawakami O., Dohmoto Y., Takenaka T. Lanthanide tetrad effects in nature: two mutually opposite, W and M // Geochem. J. 1987. V. 21. P. 119-124.

21. Byrne R.H., Li B. Comparative complexation behavior of the rare earth // Geochim. Cosmochim Acta. 1995. V. 59. № 22. P. 4575-4589. 